Revue des patrimoines

25 | 2014

Le patrimoine de la Grande Guerre

\title{
Une base de données sur les monuments aux morts : histoire concrète et valorisation numérique
}

Martine Aubry and Matthieu de Oliveira

\section{(2) OpenEdition}

\section{Journals}

Electronic version

URL: http://journals.openedition.org/insitu/11551

DOI: 10.4000/insitu. 11551

ISSN: 1630-7305

Publisher

Ministère de la Culture

\section{Electronic reference}

Martine Aubry and Matthieu de Oliveira, « Une base de données sur les monuments aux morts

histoire concrète et valorisation numérique », In Situ [Online], 25 | 2014, Online since 30 January 2015,

connection on 01 July 2020. URL : http://journals.openedition.org/insitu/11551 ; DOI : https://doi.org/ 10.4000/insitu. 11551

This text was automatically generated on 1 July 2020 .

\section{c) (7) $९$}

In Situ Revues des patrimoines est mis à disposition selon les termes de la licence Creative Commons Attribution - Pas d'Utilisation Commerciale - Pas de Modification 4.0 International. 


\title{
Une base de données sur les monuments aux morts : histoire concrète et valorisation numérique
}

\author{
Martine Aubry and Matthieu de Oliveira
}

1 «Passant, souviens-toi! » ${ }^{1}$. Dans un pays comme la France, une telle injonction ne manque pas d'être réitérée presque à chaque coin de rue de chaque commune. Elle vise à entretenir le souvenir de ceux qui ont fait le sacrifice de leur vie pour le pays et se trouve matérialisée dans une multiplicité de monuments commémoratifs. Le plus visible, le plus fréquent est sans conteste le monument aux morts, symbole des conflits passés et rappel des morts pour la patrie, périodiquement célébrés lors de cérémonies officielles du 11 Novembre. Avec le décès, le 12 mars 2008, de Lazare Ponticelli, dernier poilu survivant, sans doute leur portée mémorielle s'en trouve-t-elle accrue puisque cette disparition a fait définitivement basculer la Grande Guerre de l'ère des témoins à celle de l'histoire.

On distingue principalement deux types de monuments : d'une part les mémoriaux, à dimension nationale et élevés sur les champs de bataille comme à Douaumont (Meuse), où est installé l'Ossuaire national inauguré par le président Lebrun en 1932, à NotreDame-de-Lorette ou à Vimy (Pas-de-Calais), qui abrite le Mémorial canadien, mémoriaux le plus souvent couplés avec des cimetières militaires; d'autre part les cénotaphes collectifs et à vocation plus locale, qui nous occuperont ici en ce qu'ils sont les plus répandus.

3 Il suffit en effet de déambuler dans les rues d'un village ou d'une ville pour rencontrer un ou plusieurs de ces édifices commémoratifs, morceaux d'histoire encore à vif, mêlant intimement histoire individuelle et collective, locale, nationale et internationale. Ils sont autant de "tableaux d'honneur destinés à proclamer les noms de ceux qui sont tombés au champ d'honneur " selon les termes d'Annette Becker. Véritable archive à ciel ouvert, placée sous le regard de chacun, ils font l'objet de l'attention des historiens professionnels et amateurs depuis un peu plus de trente ans 
et ont trouvé leur place dans l'historiographie contemporaine de la guerre et de ses représentations.

Depuis 2006, une équipe d'historiens de l'université de Lille $3^{2}$ s'est mobilisée pour mener à bien une enquête à vocation tout d'abord départementale puis régionale. Disposant bientôt d'une masse considérable d'informations, les enseignants-chercheurs de l'Institut de Recherches Historique du Septentrion (IRHiS - UMR CNRS 8529) ont opté pour la mise sur pied d'une base de données leur permettant de clairement visualiser les «blancs sur la carte» et de traiter systématiquement les informations recueillies à partir d'un questionnaire précis. Les nouvelles technologies offrent en effet la possibilité de fédérer les initiatives éparses et de rassembler en un seul lieu, virtuel, l'ensemble des informations disponibles, tout en permettant, au prix d'un effort d'approfondissement et d'uniformisation des données, des requêtes croisées.

Les commémorations du centenaire de la Première Guerre mondiale ont constitué, on le comprend aisément, une occasion de donner à cette initiative une ampleur plus large tout en permettant des comparaisons à l'échelle du pays tout entier. Les éléments recueillis aux échelles locales, régionales et/ou nationales permettent dès lors de confronter les conclusions des travaux jusque-là disponibles avec les informations géographiques, physiques et archivistiques disponibles et réunies au sein d'une base de données nationale, labellisée par la Mission du centenaire en 2013.

Dans sa configuration actuelle, la base de données consacrée aux monuments aux morts abritée par l'IRHiS couvre la quasi-totalité de la France métropolitaine ${ }^{3}$. Elle recense à ce jour près de 2500 monuments en France (sans compter 11 monuments belges), même si des biais méthodologiques sont à signaler : à ce jour, plus des trois quarts de ces monuments sont situés dans le Nord (913) et le Pas-de-Calais (922), près des neuf dixièmes dans la moitié septentrionale du pays, seules les régions Rhône-Alpes (65 monuments) et Auvergne (61 monuments) allant au-delà d'une présence parfois symbolique. Ce chiffre est à mettre en regard des 36683 communes françaises (actuelles) et permet de mesurer le chemin qui reste à parcourir pour couvrir l'ensemble du territoire, DOM et TOM inclus, d'ici au centenaire de l'armistice de 1918.

7 Dans la plupart des cas, il n'existe qu'un seul monument aux morts par commune et de fait, la base recense 1989 «monuments communaux », soit plus de $80 \%$ du total. Pour autant, certaines villes, et les plus importantes d'entre elles au premier chef, peuvent en compter plusieurs, ne serait-ce qu'au titre des quartiers ou des arrondissements. On en trouve par exemple 8 dans la capitale et 3 à Lyon, et la liste est très loin d'être exhaustive. Mais là encore, les résultats demeurent très lacunaires et demandent à être complétés peu à peu, grâce à une large mobilisation des équipes universitaires comme des passionnés, organisés ou non en sociétés savantes et autres clubs de généalogie.

La souplesse d'utilisation de la base de données, en particulier via la fonction " dépôt ", permet à chacun d'apporter sa pierre à l'édifice et de constater en temps réel l'avancée des travaux. Cette facilité d'usage n'a d'égale que la rigueur du questionnaire mis sur pied par l'IRHiS et qui seule permet de disposer d'informations nombreuses et complètes, tirées du monument lui-même comme de la presse d'époque et/ou des archives (communales et départementales). Tels sont les éléments mis en lumière dans la dernière partie de ce texte qui présente les fonctionnalités et autres règles de fonctionnement de ce formidable outil de recherche évolutif et collaboratif. Cette entreprise n'aurait bien évidemment pas pu être réalisée sans qu'aient été mobilisés les travaux publiés depuis une trentaine d'années et qui font l'objet d'un état des lieux en 
ouverture de cet article. La partie centrale du texte est enfin consacrée à la présentation des résultats, provisoires mais encourageants, d'une première enquête régionale centrée sur le Nord-Pas-de-Calais.

\section{Un tour d'horizon historiographique}

9 Le premier à s'être véritablement saisi de la question est Antoine Prost qui, dans sa thèse sur Les Anciens combattants et la société française 1914-19394, s'est livré à la première enquête sur les monuments aux morts : il estime alors leur nombre à 38000 , ce qui placerait le corpus réuni par la base de données à environ $6,5 \%$ de ce chiffre de départ.

Il établit également une première typologie, selon la forme générale, l'ornementation, l'épigraphie ou encore l'emplacement ${ }^{5}$. Ces travaux pionniers ont été successivement prolongés par les synthèses de Philippe Rivé et d'Annette Becker, qui y voit une bonne illustration de la "Mémoire de la Grande Guerre " ${ }^{6}$ : entretenant la mémoire des morts afin que celle-ci prenne un sens, ils représentent le plus souvent des soldats et célèbrent selon les cas la Victoire, la Liberté, la République ou encore la Paix. Symboles de douleur et/ou de ferveur, ils portent très exceptionnellement un discours pacifiste ${ }^{7}$ et témoignent avant tout du déroulement de la guerre et de l'état d'esprit des survivants.

11 Apparus massivement au lendemain de la Première Guerre mondiale, ces monuments s'inscrivent dans la continuité de ceux qu'on a vu apparaître à la suite de la guerre de $1870^{8}$. Ces derniers datent le plus souvent des années 1890 et s'inscrivent alors dans un contexte particulier : il demeure en effet bien difficile de célébrer une défaite militaire et c'est plutôt la revanche que l'on prépare en entretenant le souvenir. Ils demeurent dès lors assez rares - la base de données en recense à l'heure actuelle environ 250 - et bien souvent liés à un épisode local considéré comme particulièrement glorieux9

12 Au lendemain de la victoire de 1918, le mouvement est massif, mêmes si les motivations sont plurielles : les Français désirent à la fois célébrer la paix et la victoire tout autant que commémorer les disparus, comme pour donner un sens à la terrible saignée (on compte 1,45 million de morts, auxquels il faut encore ajouter 1,1 million d'invalides de guerre) qu'a connue un pays déjà affaibli sur le plan démographique ${ }^{10}$. Ces différents éléments expliquent qu'un consensus se fasse rapidement pour donner une dimension nationale aux hommages prévus. En quelques années, trois lois précisent la façon dont l'État prévoit d'apporter son soutien à ces commémorations :

- la loi du 25 octobre 1919 sur la « commémoration et la glorification des morts pour la France au cours de la Grande Guerre » pose le principe d'une subvention publique « en proportion des sacrifices consentis ";

- la loi de finances du 31 janvier 1920 fixe les modalités précises du soutien financier de l'état ;

- la loi du 24 octobre 1922 enfin fait du 11 novembre une fête nationale ${ }^{11}$.

13 Cet engouement législatif se trouve prolongé sur le terrain par l'érection des premiers monuments aux morts dès 1920, relayée par leur inauguration dès 1921 dans les villages, le mouvement se poursuivant jusque dans les années 1930 en ville, où les enjeux mémoriels, le nombre des morts mais aussi des monuments sont souvent multiples. Dans tous les cas, il s'agit du résultat d'un long processus, renvoyant à une réalité complexe. 

débattre et opérer des choix : si chacun partage la volonté d'honorer les morts de la commune, les lignes de partage peuvent exister entre les individus, les groupes d'affinités ou les partis et les procès-verbaux des réunions du conseil municipal sont parfois tendus et imposent des retards, du moins des arbitrages. Les débats portent le plus souvent sur la forme ou la nature du monument, le texte de l'inscription qui y sera gravé et plus largement, le message que l'on entend véhiculer ${ }^{12}$. Mais c'est surtout sa localisation qui déchaîne les passions, opposant les tenants d'une vision républicaine ou laïque - qui militent en faveur d'une implantation sur la place ou à proximité immédiate de la mairie à ceux qui voudraient l'installer à l'ombre de l'église, voire dans le cimetière, tant et si bien que certaines petites communes disposent de plusieurs monuments concurrents.

gros sujet de débat reste le financement, qui associe le plus souvent fonds gouvernementaux et communaux, parfois publics et privés (sous la forme d'une éventuelle souscription). Pour partie liée au nombre de morts, ils suscitent parfois une sordide concurrence des malheurs ${ }^{13}$. Et une fois la somme disponible, il ne reste plus qu'à faire son choix parmi les multiples options proposées par les entrepreneurs qui se sont habilement positionnés sur ce marché commercial particulièrement rentable ${ }^{14}$. Le catalogue des Marbreries générales Gourdon, installées à Paris, propose ainsi en décembre 1921 un large éventail de produits destinés à orner le futur monument aux morts. En fonction des besoins et des ressources financières de la commune, elles peuvent ainsi lui fournir un "panneau d'inscription pouvant recevoir 400 noms » de $3 \mathrm{~m}$ de hauteur pour une somme allant de $5500 \mathrm{~F}$ (en marbre) à $1840 \mathrm{~F}$ (en pierre et granit silicaté) ; une statue de «Poilu combattant » de $1,95 \mathrm{~m}$ de haut coûte $13400 \mathrm{~F}$ en granit, $15900 \mathrm{~F}$ en bronze, $11900 \mathrm{~F}$ en marbre, mais seulement $2350 \mathrm{~F}$ en pierre silicatée; de son côté, le très demandé "Poilu sentinelle » $(2 \mathrm{~m})$ ne se vend qu'en marbre, pour $10800 \mathrm{~F}$; encore faut-il y ajouter quelques ornements tels qu'une « croix de guerre » (4 800 à $1560 \mathrm{~F})$, une « palme avec couronne » (900 à $180 \mathrm{~F})$, sans oublier les chaînes destinées à entourer le monument ( $50 \mathrm{~F}$ le m).

Vient enfin le moment de la communion collective des habitants, lors de l'inauguration du monument aux morts, en présence des anciens combattants, des civils et des enfants ${ }^{15}$. Le 3 juin 1934, celui de Bouvines (Nord), qui compte 16 noms, est inauguré en présence de trente associations d'anciens combattants, de sociétés de musique, de gymnastique, compagnies de sapeurs-pompiers, entourés par une foule estimée à 8000 à 10000 personnes.

17 Il existe pourtant quelques rares communes dans lesquelles on ne trouve pas de monument aux morts. Elles représenteraient selon A. Prost environ $1 \%$ seulement des communes, ce qui porterait toutefois leur nombre à plus de 350. La littérature disponible sur le sujet, bien peu scientifique dès lors qu'il s'agit d'articles de journaux le plus souvent régionaux ou locaux - et de magazines, n'en recense pourtant que moins d'une dizaine, en toute logique inégalement répartie sur l'ensemble du territoire national ${ }^{16}$.

18 Cette absence peut s'expliquer par trois raisons au moins :

- il s'agit en premier lieu d'une affaire de statistique, dès lors que ces communes étaient ellesmêmes en train de disparaître en raison de la désertification, comptant majoritairement des habitants hors d'état de combattre (personnes âgées, femmes, pas ou peu d'enfants), et viennent alors à l'esprit des régions rurales et/ou de montagne, alors même que le déclin 
démographique s'est amorcé dès la fin du XIX siècle. Et de fait, chacune des communes repérées comptait moins de 200 habitants à la veille du conflit - et au moins deux moins de 100 -, ce qui limite statistiquement le risque de voir un des rares mobilisés de la commune « mourir pour le France »;

- vient ensuite la chance, puisque tous les « enfants » du village mobilisés - leur nombre n'est pas toujours connu avec précision - sont rentrés vivants de leur passage au front, sans doute au moins une fois en première ligne, et ayant fait preuve d'un héroïsme « raisonnable ${ }^{17}$. Ce qui ne veut pas dire qu'ils en soient revenus indemnes, physiquement ou psychologiquement ${ }^{18}$;

- dernier cas de figure, elles n'existaient pas/plus au moment de la Première Guerre mondiale ; c'est par exemple le cas de deux des 34 communes de Martinique, Sainte-Lucie et Saint-Pierre, rayées de la carte par la catastrophe de la Montagne Pelée de 1904 et recréées en $1923^{19}$.

Pour autant, l'absence de monument aux morts n'exclut pas ces communes des commémorations nationales: tous les trois ans, celle de Palazingues (Corrèze) s'acquitte du prix des couronnes déposées chaque 11 Novembre au pied du monument aux morts de sa voisine Aubazine. Et à Beuzeville-au-Plain (Manche), on peut se recueillir devant une stèle érigée en mémoire des 22 soldats états-uniens décédés lors des opérations du débarquement de juin 1944.

En toute logique, il conviendrait également d'analyser le cas des villes et villages qui ont certes compté eux aussi des "morts pour la France", mais qui ont physiquement disparu du fait même des combats. C'est le cas de quelques communes de l'Aisne Ailles, Beaulne-et-Chivy, Courtecon et Vauclerc-et-la-Vallée-Foulon -, totalement détruits à la suite des combats d'avril 1917 sur le Chemin des Dames. Leur territoire, classé en zone non constructible (la « zone rouge »), a été administrativement rattaché à celui d'une commune voisine au lendemain du conflit. Quelques autres - Cerny-enLaonnois, Craonne, Allemant - ont été reconstruits à quelque distance, sans perdre leur existence administrative. Et le cas se répète également dans la Meurthe-et-Moselle, la Meuse ou la Marne. Une étude approfondie permettrait de rendre compte de la situation particulière de ces "villages disparus ", dont les monuments aux morts font figure de « curiosité ».

21 L'impression générale porte enfin à considérer que les monuments sont «tous pareils »; pourtant aucun n'est identique à l'autre. Une étude précise et comparative permet de distinguer, sans doute assez rapidement, des modèles régionaux ou cantonaux, des messages variables ou divergents. L'on peut ainsi observer la façon dont les uns et les autres ont mobilisé les signes extérieurs de la victoire, de la gloire, de la paix, de la perte et de la douleur.

22 C'est en tout cas ce que les synthèses et autres recherches menées à l'échelle départementale ont permis de confirmer ou d'affiner. On dispose ainsi de travaux, pour certains contemporains des travaux d'A. Prost, qui couvrent tout ou partie des départements des régions du grand $E^{2}{ }^{20}$, du quart Sud-Est ${ }^{21}$, du Centre ${ }^{22}$ et du SudOuest $^{23}$ et d'ailleurs encore ${ }^{24}$, même si les blancs sur la carte sont encore légion. Plus près de Lille, il existe enfin des travaux consacrés à l'Aisne ${ }^{25}$ et à la Somme ${ }^{26}$.

23 Pour autant et jusqu'à ce que la base de données abritée par l'IRHiS soit plus amplement complétée, la comparaison terme à terme des différents éléments constitutifs d'un monument aux morts s'avère bien souvent difficile. 


\section{Mesurer la spécificité du Nord-Pas-de-Calais}

En revanche, le programme de recherche initié par l'IRHiS attribue de fait à la région Nord-Pas-de-Calais un rôle de terrain d'expérimentation privilégié, et d'abord en raison même de sa situation militaire ${ }^{27}$. La région présente entre autres la particularité d'avoir été pendant presque tout le conflit une zone de combat et d'apparaître ainsi au premier rang des zones touchées par les destructions ${ }^{28}$. Parallèlement, ses habitants ont vécu, selon leur lieu de résidence, des situations très différentes les unes des autres puisque si l'on va d'ouest en est, on voit se succéder l'arrière du front, les lignes alliées, le front, les lignées allemandes et enfin le territoire occupé 29.

Ces situations ont à l'évidence des répercussions sur les monuments aux morts. En premier lieu, parce que le Nord et le Pas-de-Calais se trouvent pour partie occupés, les monuments commémoratifs tiennent également compte du cas de civils, otages et/ou exécutés, dont les noms apparaissent aux côtés des soldats «tombés pour la France " ${ }^{30}$. La région présente enfin des particularités à la fois historiques et culturelles qui font une partie de sa spécificité. On note ainsi des différences notables dans les activités économiques (agricoles ou industrielles), la pratique religieuse (la Flandre maritime plus catholique que le Valenciennois) ou encore le «tempérament» politique (du nationalisme conservateur, fréquent en situation frontalière, au socialisme radical) ${ }^{31}$. Ce sont tous ces éléments qui se retrouvent, de façon plus ou moins affirmée ou visible, sur les monuments aux morts nordistes et qu'il convient de décrypter en mettant à profit les possibilités offertes par la base de données, en particulier la multiplicité de requêtes - éventuellement croisées - qu'elle rend possible et la géolocalisation - parfois très fine - des résultats sur une carte.

La localisation des monuments sur le territoire de la commune constitue à cet égard un premier angle d'attaque: dans près de $60 \%$ des cas, la dimension religieuse semble privilégiée avec $30 \%$ des monuments situés "près de l'église", un quart dans le cimetière et de façon plus résiduelle (4\%) dans l'église même. À l'inverse, les monuments "laïcs" se retrouvent dans un peu plus de $40 \%$ des cas, les places (un tiers) devançant de beaucoup les rues (7 \% environ). Si la géographie ne permet pas de faire apparaître de modèle clair qui opposerait par exemple l'est et l'ouest de la région, c'est bien souvent que les communes comptent plusieurs monuments, qui répondent donc aux attentes de ceux qui les ont érigés, non sans soulever d'autres questions. On compte en effet 916 et 922 monuments pour 650 et 895 communes dans le Nord et le Pas-de-Calais : une simple constatation arithmétique permet d'affirmer qu'il y a donc, en moyenne, plus d'un monument par commune, avec une forte prime pour le Nord (1,4 monument par commune). C'est par exemple le cas de Viesly, commune du Cambrésis, forte de 2421 habitants lors du recensement de 1911 et qui ne compte pas moins de 3 monuments aux morts, dans 3 endroits différents de son territoire et commémorant pour partie des conflits différents. Le premier, situé près de l'église, rappelle le souvenir des morts de la guerre de 1870 ; le second, sur la place NarcissePavot, rend hommage aux combattants de la Première Guerre mondiale ainsi qu'à l'unique Vieslysien décédé en Afrique du Nord; le troisième enfin est le monument paroissial de l'église Saint-Martin, qui liste les morts de la Première comme de la Seconde Guerre mondiale sans oublier la plaque consacrée au sous-lieutenant Havret " massacré en Indochine ", en 1945 de surcroît. Pour ajouter encore à la confusion, la liste des noms de ces « morts pour la France » varie d'un monument à l'autre ${ }^{32}$. 
27 À l'inverse, on recense quelques rares communes sans monument, soit qu'il n'y en ait jamais eu - et la liste présentée supra se trouve de fait augmentée -, soit que les relevés n'aient pas permis, à ce jour, de le localiser. Le monument a en effet pu être détruit, déplacé, démonté, oublié... Dans certains cas sans doute assez rares, le monument est commun à deux communes, comme celui que se partagent Estrées et Estréelles (Pas-deCalais), situé sur la route principale, en lisière des deux villages. Si la dédicace est unique - «Aux enfants d'Estrées et Estréelles morts pour la France 1914-1918» -, chaque côté du monument est dédié à une commune.

Si l'on s'intéresse ensuite au projet architectural retenu pour illustrer les monuments régionaux, on voit se confirmer sans réelle surprise le grand succès du " poilu ». Cette figure emblématique est sans conteste la plus représentée, ici comme ailleurs en France (plus de 690 occurrences dans la base à ce jour), dont 310 dans le Nord et 248 dans le Pas-de-Calais. Dans ces deux départements et en dépit d'une surreprésentation évidente (avec leur présence sur $34 \%$ et $27 \%$ des monuments), on remarque une répartition géographique bien loin d'être uniforme puisque cet élément de la statuaire commémorative est très majoritairement repérable dans la partie orientale du Nord et le sud du Pas-de-Calais. Or il s'agit justement de celles qui ont subi l'occupation allemande pendant la majeure partie du conflit. Si l'on affine la requête en recherchant spécifiquement les " poilus sentinelles ", on en trouve 90 dont 45 dans le Nord et autant dans le Pas-de-Calais, là encore très majoritairement repérés dans ces mêmes arrondissements orientaux et méridionaux (41 sur 45 dans le Nord; 22 sur 45 dans le Pas-de-Calais), comme pour réaffirmer dans la pierre la présence française sur une terre qui a vu tant de combats. Une requête portant sur leurs alter ego "mourant " donne des résultats comparables avec 55 et 42 occurrences, essentiellement localisés dans le Cambrésis, alors que les " poilus grenadiers » (respectivement 7 et 6) sont quant à eux presque exclusivement repérables le long de l'ancienne ligne de front. Là encore, la prégnance du conflit, par-delà la cessation des hostilités, se trouve sans cesse rappelée.

La figuration du coq constitue également un "classique » des monuments aux morts tant il est immédiatement lu comme un symbole national dans l'imaginaire collectif. On en retrouve certes plusieurs dizaines (77 dans le Nord et 44 dans le Pas-de-Calais), mais leur présence apparaît comme plus ponctuelle que véritablement illustrative d'un nationalisme exacerbé. Plus significative, ne serait-ce qu'en termes quantitatifs, la croix latine apparaît à 975 occurrences (434 dans le Nord et 541 dans le Pas-de-Calais), soit dans plus de $60 \%$ des monuments régionaux et presque uniformément répartis, du moins apparemment. Leur densité est en effet sensiblement plus importante dans la partie occidentale de la région, à l'ouest d'une ligne Bourbourg-Arras, dans cette Flandre farouchement catholique étudiée en son temps par Yves-Marie Hilaire ${ }^{33}$.

La chronologie fine des inaugurations permet également de préciser ce qui ne relevait jusque-là que de l'hypothèse ou de la constatation locale. Les résultats proposés ici ne sont cependant que très partiels, la date précise de l'inauguration n'étant connue que dans moins de 400 communes (sur 1545). Logiquement, la décennie 1919-1939 arrive très largement en tête avec respectivement 108 occurrences dans le Nord (16,6 \% des communes) et 229 dans le Pas-de-Calais (25,5\%). Et c'est dès 1919 qu'ont lieu les premières inaugurations, dans 5 communes de la région (4 du Pas-de-Calais et une du Nord $)^{34}$, la plus précoce étant celle de Blangy-sur-Ternoise qui procède à la cérémonie dès le 14 juillet suivant l'armistice. Par la suite, les cérémonies se font beaucoup plus 
rares: 12 entre 1930 et 1939; aucune pendant l'Occupation; 3 entre 1945 et 1960, souvent en lien avec la « réactivation » du monument au lendemain de la Libération; aucune entre 1961 et 1975 ; 3 entre 1976 et 1990 ; enfin 7 entre 1991 et 2013 . Et encore, dans le dernier cas de figure, s'agit-il le plus souvent de ré-inauguration du monument de la Première Guerre mondiale, comme à Mérignies (Nord). Le monument original, commandé en 1920, est inauguré l'année suivante ; ce n'est qu'au début du XxI ${ }^{\mathrm{e}}$ siècle qu'il est déplacé puis restauré avant d'être finalement inauguré de nouveau le 11 novembre 2009. À cette occasion, le maire rappelle qu' « en perdant 24 hommes pour une population de 805 habitants, notre village n'a pas échappé à cette guerre totale. Parmi les victimes on dénombre notamment 4 cultivateurs, 6 ouvriers agricoles, 1 employé des chemins de fer, 1 cordonnier et 1 meunier. "

31 Enfin, les monuments aux morts de la Première Guerre mondiale constituent le point de convergence de la commémoration communale. Ils sont un moyen de percevoir la façon dont les habitants de la région appréhendent les conflits contemporains et entretiennent la mémoire de leurs morts ${ }^{35}$. Au-delà du cas de la guerre francoprussienne déjà évoqué, les opérations lointaines qui ont fait la «gloire de la plus grande France » trouvent également leur place dans la mémoire locale : le monument aux morts de Wallers-Aremberg (Nord) mentionne ainsi les noms de soldats morts au Tonkin en 1892, au Soudan en 1894 ou à Madagascar en 1895. Après la victoire de 1918 et tout au long $\mathrm{du} \mathrm{xx}^{\mathrm{e}}$ siècle, on voit se greffer, ici comme ailleurs, sur le cénotaphe «originel » toute une série de plaques commémorant les soldats tombés lors de la Seconde Guerre mondiale (1 197 occurrences dans la région), des guerres de décolonisation - le plus souvent sous la dénomination d'«Indochine » (233 occurrences) et d' «AFN» (309 occurrences) -, tristes reflets de la façon dont la France métropolitaine vivait, de loin, ces conflits coloniaux ${ }^{36}$. Et sans doute verra-t-on prochainement apparaître au pied de monuments aux morts des plaques en mémoire des soldats tombés en Côte d'Ivoire, en Afghanistan, au Mali ou en Centrafrique ${ }^{37}$.

$\mathrm{Au}$ total, la France se trouve ainsi placée sous la protection de nombreux régiments de pierre ou de fonte qui veillent sur les morts et les vivants et investissent l'espace public. Ils apparaissent ainsi comme les lointains descendants des soldats des 8000 soldats de l'armée de terre cuite mise au jour lors de la découverte du mausolée de l'empereur Qin (III ${ }^{\mathrm{e}} \mathrm{s}$. avant notre ère). Et dans sa bande dessinée Zappe la guerre ${ }^{38}$, le dessinateur Pef imagine la «résurrection" des morts du monument et leur regard sur le monde d'aujourd'hui, comme pour réaffirmer la place des monuments aux morts dans la société contemporaine et les symboles dont ils sont porteurs.

\section{La base [au 4 février 2014]}

Prévue à l'origine sur l'aire géographique du Nord et du Pas-de-Calais, elle est désormais passée à une dimension nationale. La situation géographique de notre laboratoire nous a également incités à prendre en compte la Belgique. Cette nouvelle dimension n'aurait pu se faire sans l'aide de la Mission du centenaire, de l'université de Lille 3 et de collectivités régionales.

34 À ce jour, la base cartographie de façon dynamique 2491 fiches de monuments aux morts $^{39}$. Le Nord et le Pas-de-Calais en comptent à eux seuls 1838 . La collecte pour le reste de la France et la Belgique ne fait que commencer. 
en interne, faire une découverte rapide de la base et nous terminerons par un mode
d'emploi destiné aux futurs contributeurs, que nous espérons nombreux. Un espace de
contact en base de la page d'accueil vous permet de nous contacter.

\section{La méthode de travail}

\section{La grille de travail}

Définie à l'origine sous un format Filemaker, elle a été approfondie et amendée au fur et à mesure pour être mieux adaptée à l'évolution actuelle de la base collaborative (version sous PHP-MySQL) 


\begin{tabular}{|c|c|}
\hline Communes & $\begin{array}{l}\text { Dans la base en ligne (Champ Commune actuelle ou Code postal), les noms de } \\
\text { communes actuelles sont prédéfinis pour la France et la Belgique sur la base } \\
\text { des codes postaux et des codes Insee. } \\
\text { Cependant pour tenir compte des communes regroupées ou fusionnées, un } \\
\text { onglet ancien nom de commune a été créé dans la time-line du monument. La } \\
\text { recherche pour ces communes se fait via le champ Recherche par mots-clefs. } \\
\text { Une fiche commune a été créée pour étudier le contexte de celle-ci pendant la } \\
\text { guerre (Décorations, la ville pendant la guerre, le nombre et le \% de morts, le } \\
\text { nom des maires...). Cette fiche est particulièrement importante pour les } \\
\text { communes situées sur la ligne de front. Certaines ont été détruites } \\
\text { intégralement et les noms des morts civils de la Grande Guerre figurent } \\
\text { souvent sur le monument aux morts. }\end{array}$ \\
\hline Localisation & $\begin{array}{l}\text { La localisation est présente de deux façons : } \\
\text { une implantation simple, dont le choix est prédéfini (près de l'église, dans } \\
\text { l'église, cimetière, cimetière militaire, place, rue et autre) et qui permet de la } \\
\text { représenter sur la carte à l'aide de pictos simples, } \\
\text { un champ de localisation plus précis a été prévu. On peut y indiquer les noms } \\
\text { de rue ou de place, s'il y a eu transfert d'un lieu à un autre... } \\
\text { Outre la carte générale, une petite carte de géolocalisation du monument est } \\
\text { présente sur la fiche. }\end{array}$ \\
\hline Description & $\begin{array}{l}\text { Celle-ci comporte } 2 \text { niveaux: } \\
\text { une description complète « littéraire » avec le plus de précisions possible, } \\
\text { une description guidée : par mots-clefs, concernant l'architecture, la sculpture, } \\
\text { les ornements, les décorations... Cette description est dénommée Produits. }\end{array}$ \\
\hline Inscriptions & $\begin{array}{l}\text { Les inscriptions sont des renseignements sémantiques importants. } \\
\text { Celles-ci sont plus ou moins longues, quelquefois accompagnées de citations } \\
\text { littéraires }\end{array}$ \\
\hline Matériaux & $\begin{array}{l}\text { Matériaux utilisés pour la réalisation du monument, des sculptures (pierre, } \\
\text { bronze, marbre...) }\end{array}$ \\
\hline Commentaires & $\begin{array}{l}\text { Constatations ou autres informations sur le monument (modifications, } \\
\text { destructions de l'ancien, rajouts, éléments disparus...). }\end{array}$ \\
\hline $\begin{array}{l}\text { Commentaires } \\
\text { supplémentaires }\end{array}$ & $\begin{array}{l}\text { Concernant les informations sur les morts, présence de plaques au pied du } \\
\text { monument, de plaques émaillées avec portraits des soldats, erreurs } \\
\text { éventuelles. }\end{array}$ \\
\hline Économie & $\begin{array}{l}\text { Les devis, marchés de gré à gré, plans de financement (subventions, } \\
\text { souscriptions publiques, dons d'entreprises, produits de fêtes, participation } \\
\text { de l'État...), réception du monument-Prix total. } \\
\text { Un champ Commentaire sur l'économie a été ajouté pour permettre des } \\
\text { remarques spécifiques. }\end{array}$ \\
\hline
\end{tabular}




\begin{tabular}{|c|c|}
\hline Sources diverses & $\begin{array}{l}\text { Notes, Bibliographie, Sources } \\
\text { Ce champ nous permet de renvoyer aux divers sites Web qui citent le } \\
\text { monument, aux travaux historiques réalisés, aux sociétés historiques et } \\
\text { particuliers qui ont accepté de travailler avec nous. } \\
\text { Sources (références textuelles et documentation photographique avec les } \\
\text { commentaires) - Archives départementales; - Archives communales; - } \\
\text { Sources diverses (documents de presse, archives privées). }\end{array}$ \\
\hline Type de monument & $\begin{array}{l}\text { Ce champ permet de différencier les monuments communaux, paroissiaux, } \\
\text { militaires... et rend possibles des recherches ciblées. }\end{array}$ \\
\hline $\begin{array}{l}\text { Médias } \\
\text { du monument }\end{array}$ & Champ iconographique : Dépôt des images. \\
\hline Auteurs & $\begin{array}{l}\text { Les noms des architectes, sculpteurs, marbriers ou entreprises sont collectés } \\
\text { dans les documents d'archives et/ou trouvés sur les monuments lors de la } \\
\text { campagne de photographies. Ensuite, nous effectuons des recherches plus } \\
\text { approfondies sur ces auteurs pour enrichir leur fiche (archives, Web, } \\
\text { références bibliographiques, photographiques...). } \\
\text { Ils font l'objet d'un onglet et de fiches spécifiques sur la base. }\end{array}$ \\
\hline Produits & $\begin{array}{l}\text { Description guidée : par mots-clefs, concernant l'architecture, la sculpture, les } \\
\text { ornements, les décorations... }\end{array}$ \\
\hline Événements & $\begin{array}{l}\text { Cet onglet va nous permettre de retracer la vie du monument, depuis sa } \\
\text { conception lors des réunions de conseils municipaux, sa mise en œuvre (traité } \\
\text { de gré à gré, travaux, courriers divers...), à son inauguration (description de } \\
\text { la cérémonie, coupures de presse...) et aux divers déplacements, rénovation, } \\
\text { réinauguration. }\end{array}$ \\
\hline Commémorations & $\begin{array}{l}\text { Toutes les guerres pour lesquelles un nom est inscrit sur le monument lui- } \\
\text { même, ou sur les plaques présentes au pied de celui-ci. } \\
\text { Les noms des morts sont restitués comme ils sont inscrits sur le monument } \\
\text { (par ordre alphabétique, par date 1914, 1915..., avec le grade, l'âge, les } \\
\text { victimes civiles ou autres indications précises...). } \\
\text { Nous n'étudions pas les morts. Pour une recherche généalogique ou } \\
\text { prosopographique à partir de ces noms, nous renvoyons systématiquement } \\
\text { au site de MemGen qui a réalisé le travail sur «l'homme» ou à d'autres sites } \\
\text { spécifiques. }\end{array}$ \\
\hline
\end{tabular}

\section{Collecte des documents anciens}

\section{Collecte de cartes postales}

Depuis 3 ans, nous avons réuni un grand nombre de cartes postales émises à partir de l'érection du monument. En novembre, un collectionneur nous a confié pour numérisation plus de 1000 cartes postales concernant toute la France, la Belgique et même quelques pays étrangers (Italie, Allemagne...) et autorisé à les publier dans la base. 


\section{Documents d'archives} animaux (coq, aigle, etc.)]; chercher les petits détails que l'on peut remarquer en faisant le tour du monument (ex. Lewarde et Escaudain, à l'arrière de la statue, outils du mineur...).

53 - prendre les photos des noms des morts inscrits sur les diverses faces du monument, ainsi que les photos des plaques au pied du monument,

54 - faire une recherche minutieuse des signatures (architecte, sculpteur, marbrier, entreprises...) qui peuvent se trouver à divers endroits du monument (sur le socle, sur l'arrière ou le côté des statues au sommet). la géolocalisation, prendre une photo avec celui-ci. Cette localisation permet ensuite de réaliser une cartographie précise des monuments, en particulier lorsque la commune en compte plusieurs. 


\section{La base - Découverte}

56 La base est accessible directement: http://monumentsmorts.univ-lille3.fr/ ou sur le portail du Centenaire 14-18: http://monuments.centenaire.org/cartographie/

\section{La page d'accueil}
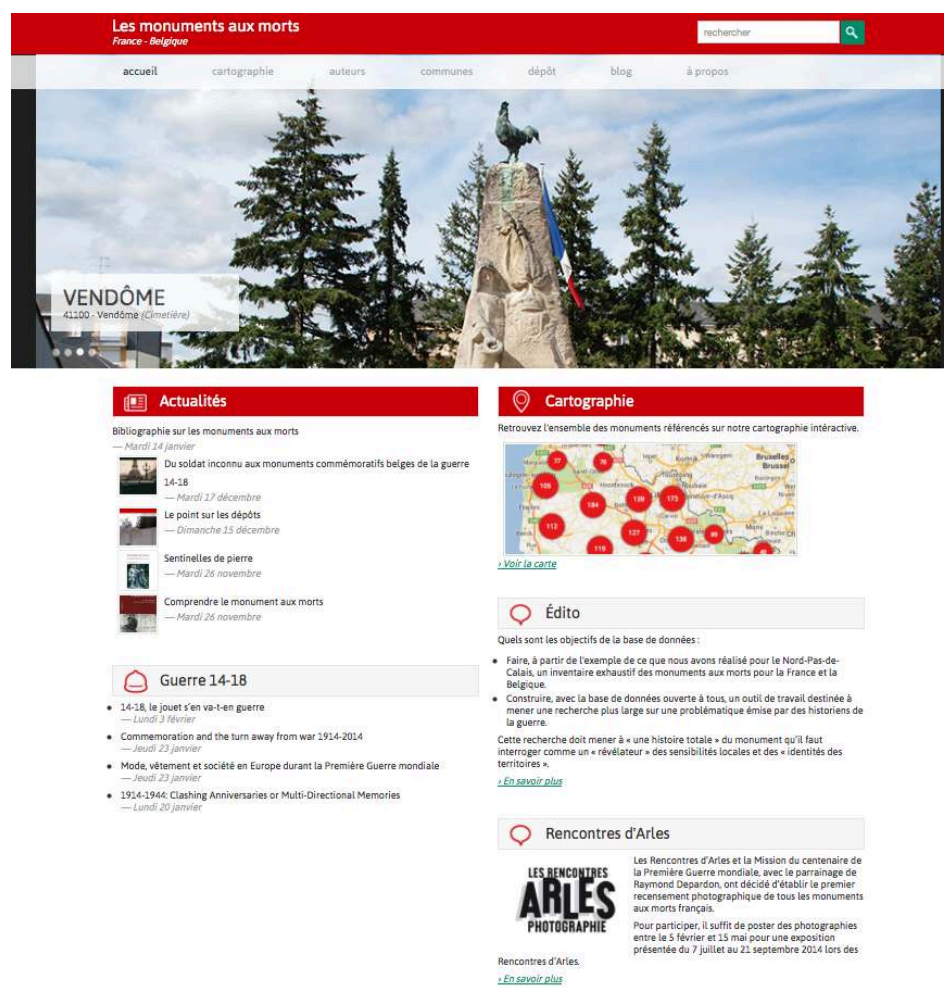

Elle comporte 7 onglets principaux et un espace correspondant au blog qui se décompose en plusieurs parties :

Actualités - Cet espace nous permet de réaliser des articles de fond, des bibliographies, des comptes rendus de livres parus sur les monuments aux morts.

Cartographie - Porte d'accès à la carte de géolocalisation des monuments. On peut y accéder également via les onglets supérieurs.

Guerre 14-18 - Vous retrouvez toutes les informations mises à jour régulièrement concernant les commémorations 14-18 (appels à communications, colloques, conférences, expositions...).

Édito - Il reprend l'historique de la base

Et pour une durée limitée, l'espace Rencontres d'Arles a été créé.

\section{Onglet Cartographie}

63 La page est divisée en 2 :

- Recherche 


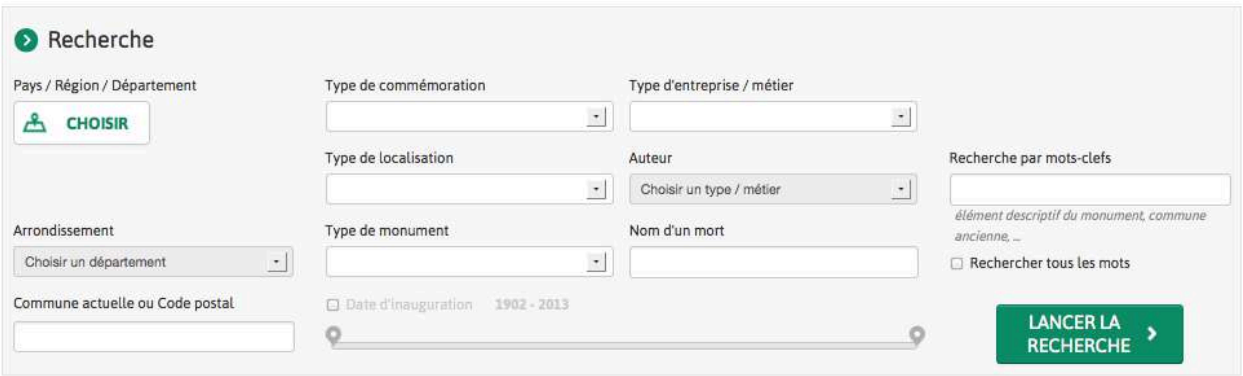

- Résultats de la recherche

- Au moyen de la carte

7) Résultats de recherche : 2491 monuments trouvés

Carte Liste

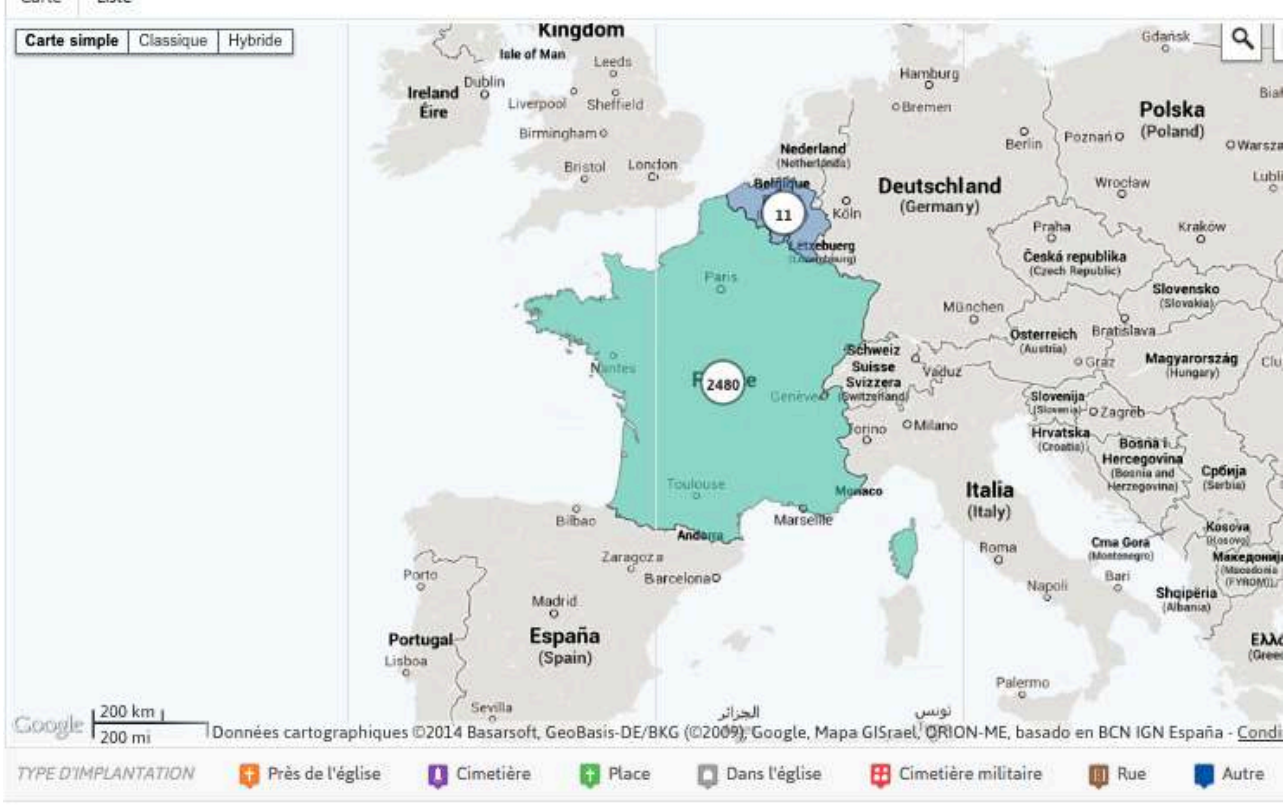

64 Sans recherche spécifique, la carte affichera la totalité des résultats, ici 2491 monuments trouvés (2 480 pour la France et 11 pour la Belgique).

En zoomant sur la carte, voici la vue au 4 février 2014 des monuments recensés. Nous avons essayé de situer au moins un monument par département, dans l'attente de nouvelles collaborations. Il reste encore beaucoup de travail pour les années à venir pour arriver en 2019 à afficher un maximum de monuments ! 


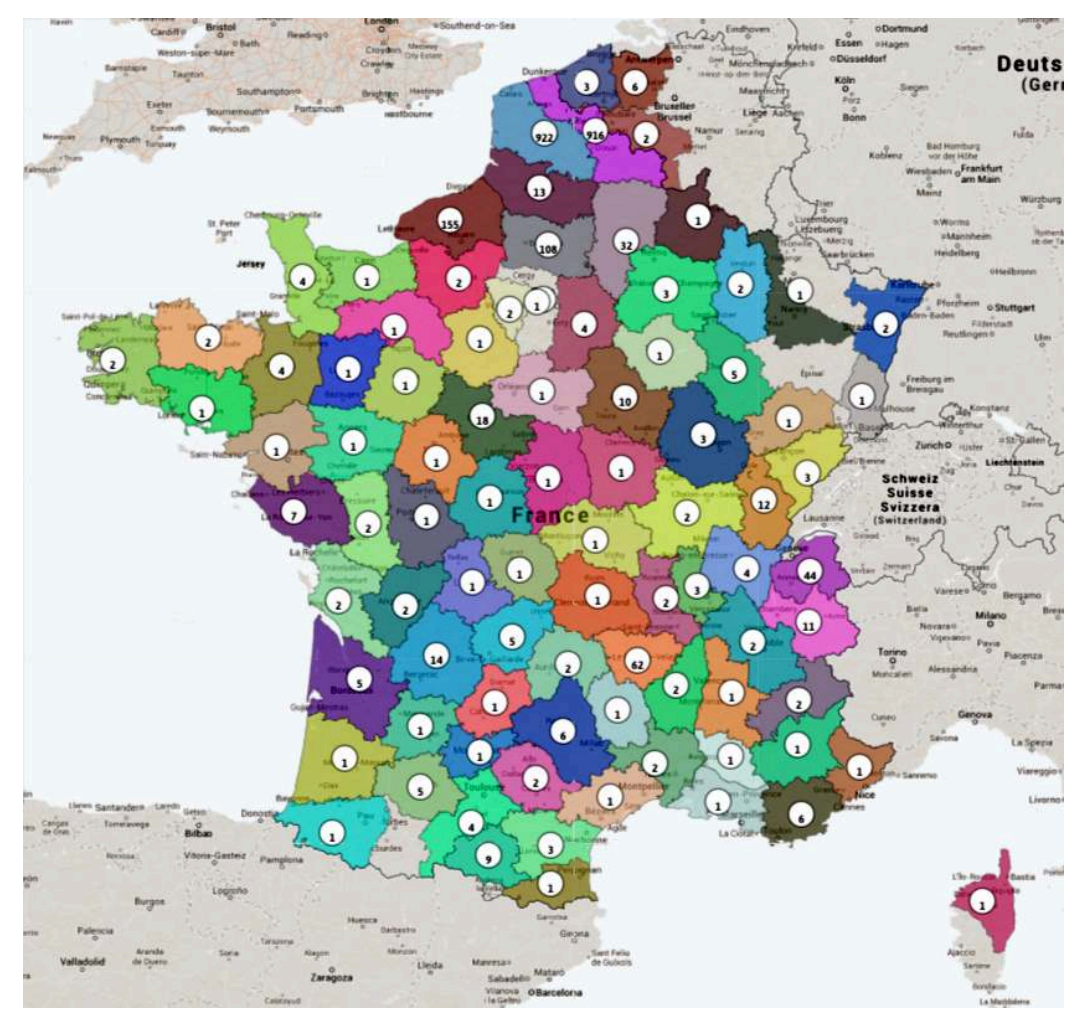

- Au moyen de l'espace Recherche

Plusieurs champs de recherche sont à la disposition du lecteur : - Géographiques : par pays, région ou département - Arrondissement ou directement par nom de commune ; - Type de commémoration; - Type de localisation; - Type de monument; - Type d'entreprise, métier...; - Auteur; Nom d'un mort (même si nous n'étudions pas les morts, on peut retrouver un nom); et enfin recherche « libre » par mots-clefs.

Une recherche a été également prévue sur la date d'inauguration.

Un exemple de recherche : Choix Seine-Maritime

- Le résultat peut s'afficher de deux façons soit sous l'onglet Carte, soit sous l'onglet Liste, comme présenté ci-dessous pour l'exemple 
(2) Résultats de recherche: 134 monuments trouvés

Carte Liste

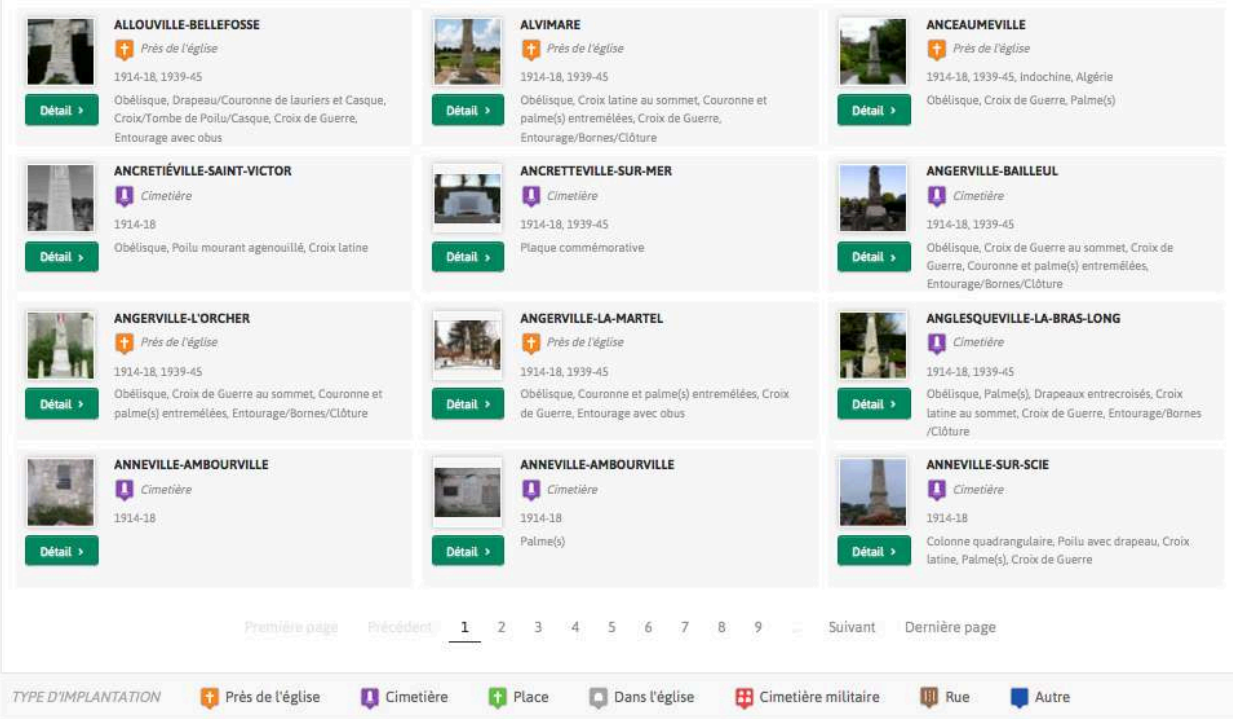

- Ensuite il ne reste plus qu'à cliquer sur détail pour accéder à la fiche complète du monument.

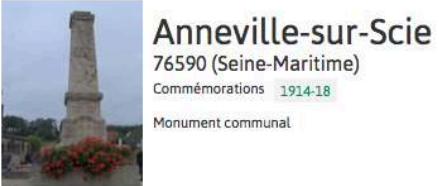

(3) Description du monument

(2) Inscriptions présentes sur le monument

(7) Les morts

(2) Auteurs / Entreprises

(2) Sources / Bibliographies / Sites Internet

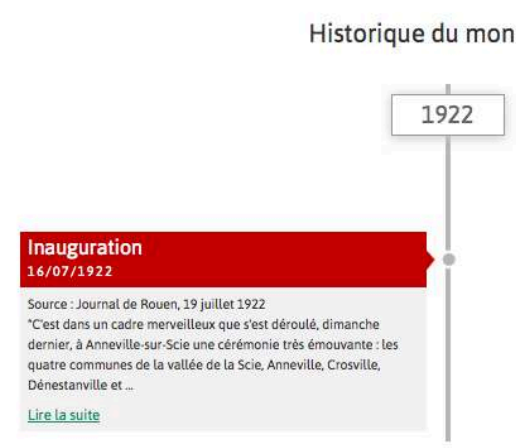

- La fiche Monument

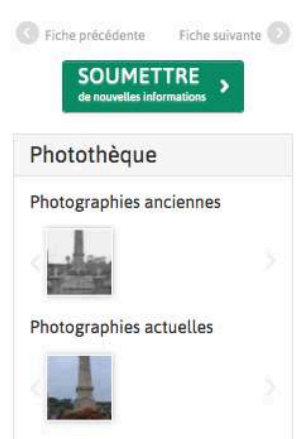

Localisation

Près de l'église Entrée du cimetière (route de Dieppe) autour de léglise

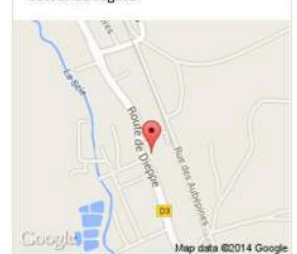

Chaque fiche est structurée de la même manière et s'appuie sur la grille définie au préalable. 


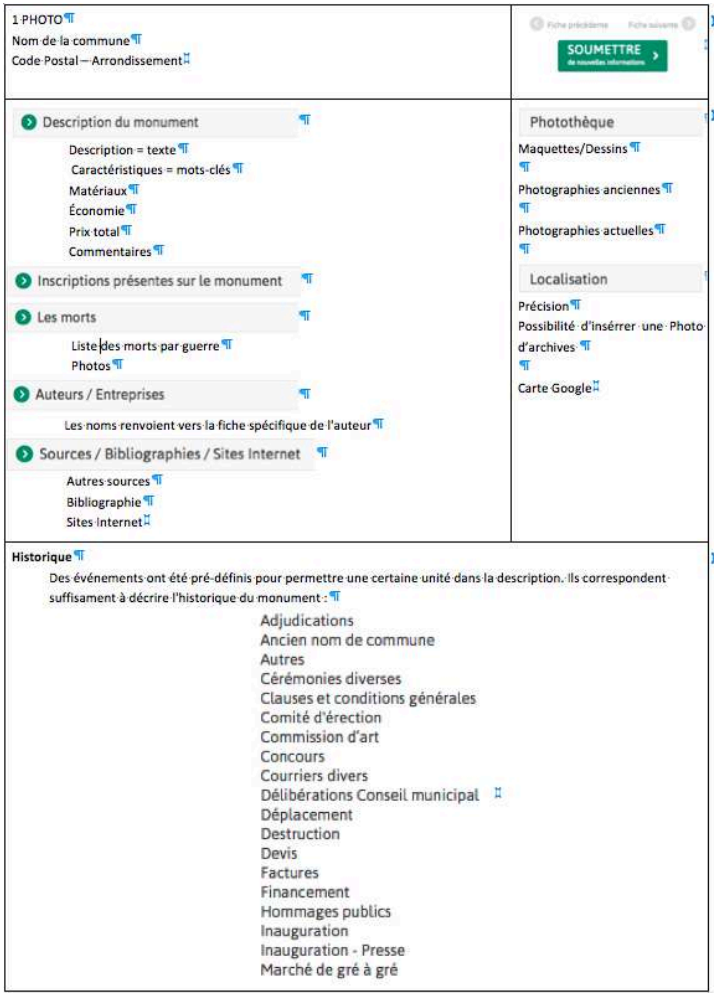

\section{Onglet Auteurs}

70 Comme pour la cartographie, un espace recherche permet de sélectionner l'auteur, par sa profession ou directement avec le nom :

\section{(2) recherche}<smiles>[Li][13CH3]</smiles>

Type d'entreprise / métier

Recherche par mots-clefs

Rechercher tous les mots

71 Par exemple, nous avons recherché CABY Charles, la fiche suivante est apparue. Il est là aussi possible de soumettre de nouvelles informations pour compléter la fiche :

CABY Charles - sculpteur

\section{- Biographie}

(1880-); né à Lille (Nord) : habite Paris Élève de Barrias, Coutan, Hypolytye Lefebre Mention honorable aux Salons des Artisties Français en 1910181 bis, rue Solterino a Litle - Vilters-Guislain : Sans appantenir vraiment aux oeuvres de série, cette figure fut diffusée à plusieurs exemplaires et ome quelques monuments aux morts. Hebuterne (Pas-de-Catais), Moncheaux (Noro). On remarquera quele est trés proche par sa composition de la statue de Bénet, editée par Ourenne, et intitulée Poilu victorieux. a sculptéte monument aux morts de

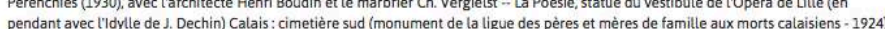

(2) CEuvres

(2) Bibliographie

(2) Liens

- Monuments dont il est l'un des auteurs

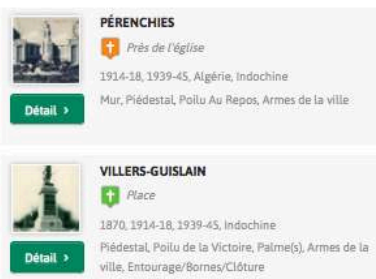

SOUMETTRE

Photothèque

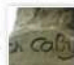




\section{Onglet Communes}

Même type de recherche possible sur une commune.

Par exemple SOUCHEZ (Pas-de-Calais), de par sa situation entre les collines de Lorette et de Vimy, a extrêmement souffert lors de la Première Guerre mondiale. Les Allemands prirent possession de la colline de Lorette dès le 5 octobre 1914 et les tentatives de reprise de ce point stratégique (la colline domine toute la plaine de Lens) par les troupes françaises échouèrent jusqu'au printemps 1915. De fait, le village fut complètement rasé. À la fiche est bien sûr relié le monument communal.

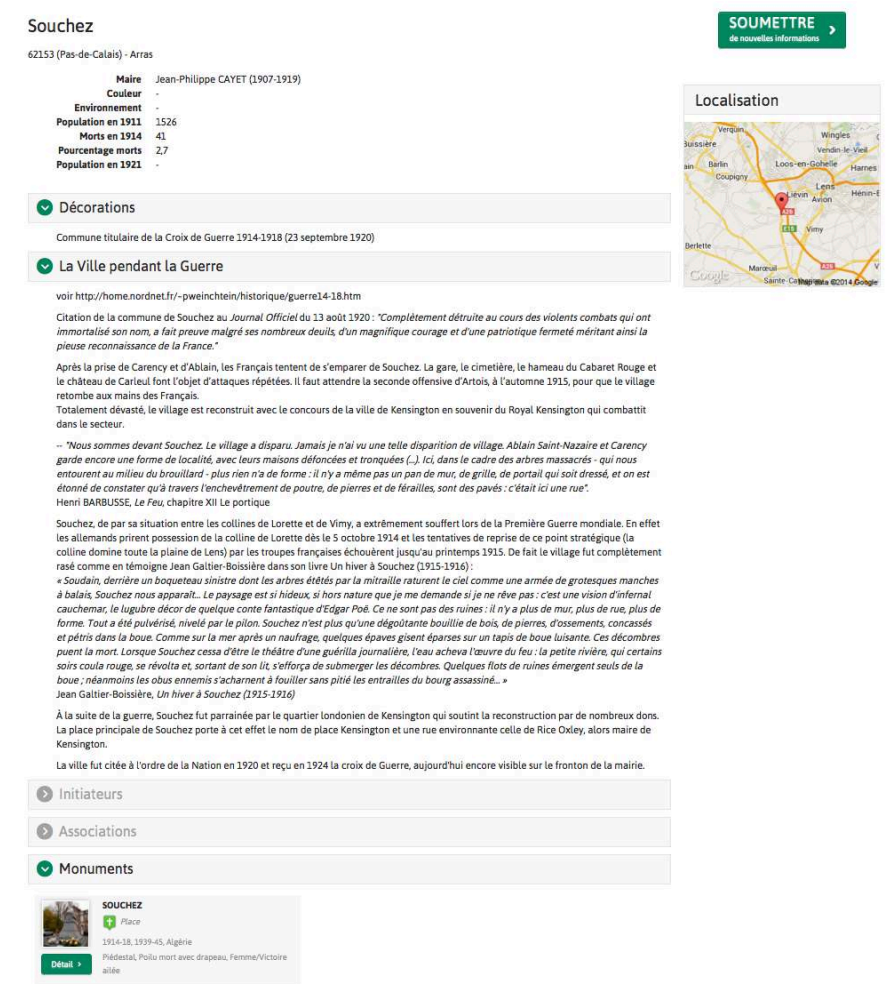

\section{La base - Mode d'emploi}

74 Depuis sa mise en ligne, le mode d'emploi de la base s'est précisé. Les diverses remarques qui nous ont été signalées par les contributeurs ont été enregistrées et ont permis certains ajustements.

\section{Onglet Dépôt}

À partir de l'onglet Dépôt, deux choix s'offrent à vous : Un dépôt complet et un dépôt rapide.

Le dépôt complet est à privilégier au dépôt rapide car il vous permet de renseigner précisément les différentes informations du monument. Le temps de validation et de publication de vos informations déposées en sera plus efficace. Nous décrirons uniquement le dépôt complet, le dépôt rapide comprend les mêmes champs.

Partie 1 

la fiche du monument pour cette commune n'existe pas déjà. Si le monument n'existe pas, vous pouvez remplir le formulaire. Devant un certain nombre de champs, vous trouverez ce symbole *. Il vous indique que la saisie est obligatoire.

79 Contact (Ces informations nous permettent d'identifier le déposant, et de pouvoir le cas échéant entrer en contact avec lui pour d'éventuelles précisions ou corrections) base.

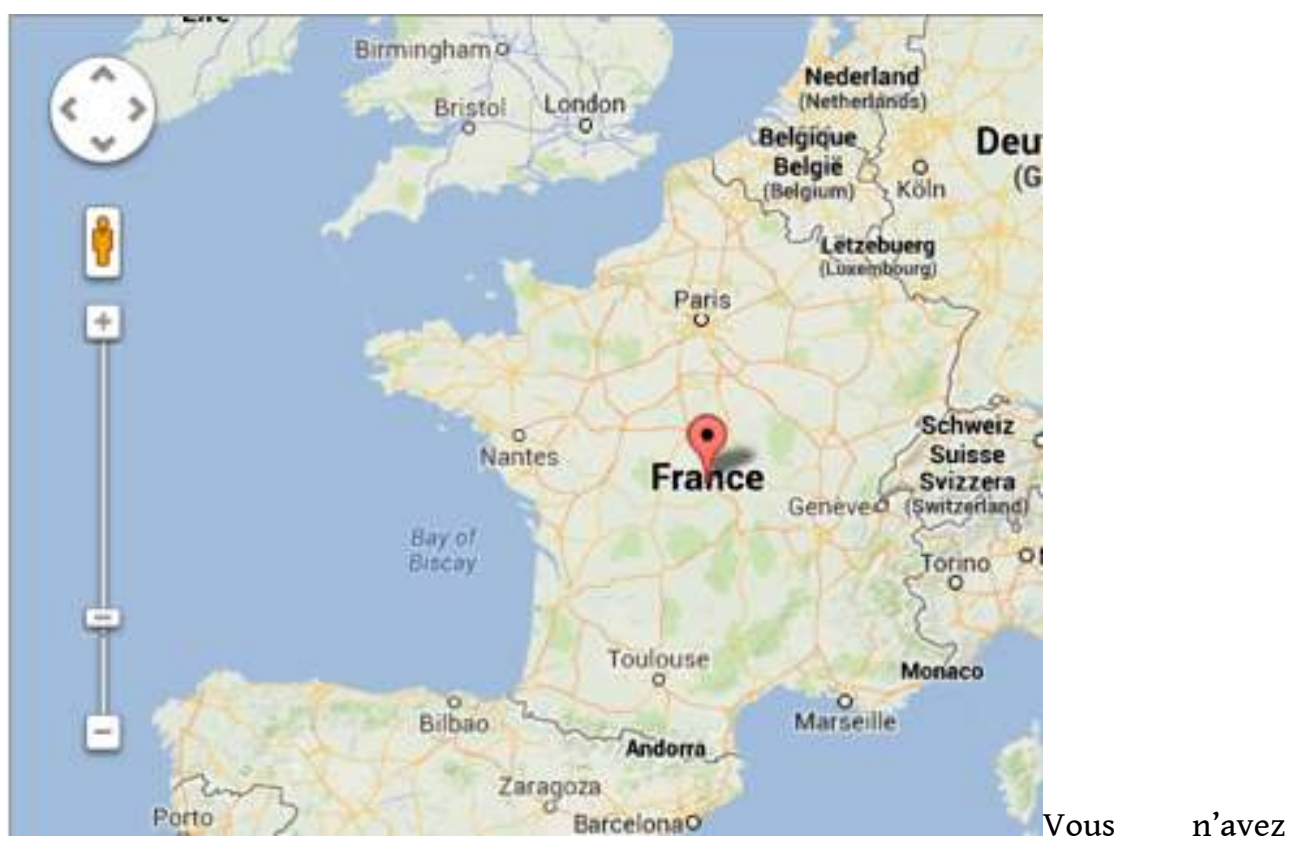

qu'à choisir celle qui vous concerne. Une fois celle-ci choisie, une géolocalisation de la commune s'affiche automatiquement.

* Type de localisation. Localisation simple et prédéfinie permettant de déterminer les pictos sur la carte. indications ou a l'aide du curseur rouge, ajuster precisement la position en déplaçant indications ou à l'aide du curseur rouge, ajuster précisément la position en déplaçant le curseur de la carte.

Les coordonnées géographiques se mettront à jour automatiquement, après avoir actualisé la carte.
* Latitude.

\section{* Longitude.}

\section{Actualiser la carte}

\section{Partie 2}

Dans cette partie, vous remplissez les zones de texte. 
Ces zones ne sont pas obligatoires : si vous n'avez rien à mettre, aucun problème (passez à la partie 3), il sera toujours temps ensuite de compléter la fiche à partir de la fiche en ligne :

\section{Informations plus précises sur la localisation du monument}

Vous précisez en indiquant le nom de la place, de la rue. La localisation d'origine du monument, s'il y a eu déplacement et bien sûr le nouveau lieu. Éventuellement le nom de la commune ancienne.

\section{Description complémentaire du monument}

Ici on peut faire une description complète ou insérer un texte ayant fait une description plus « littéraire » du monument.

\section{Inscriptions présentes sur le monument}

Souvent sur la face du monument, inscription type "La commune de... à ses enfants morts pour la France... » Quelquefois d'autres inscriptions se trouvent à l'arrière (citation, ou date de l'inauguration avec les noms des maires...)

\section{Matériaux utilisés pour la construction du monument}

Pierre ou pierre pour le socle, bronze pour la statue...

\section{Commentaires}

Tout commentaire complémentaire concernant le monument du style, « 1 seul monument pour 2 communes... ».

Commentaires supplémentaires concernant les informations sur les morts

Présence de plaques au pied du monument, description de celles-ci avec le texte inscrit dessus.

\section{Économie}

À partir des sources archivistiques, on peut remplir ces champs; si vous n'avez que le prix total, c'est déjà très intéressant.

La zone "Commentaires sur l'économie » vous permet de remplir des indications que vous pourriez avoir en dehors ou en complément des données chiffrées.

Sources diverses n'entrant pas dans le champ «Événements »

Il peut s'agir de sources connexes en relation souvent indirectes avec le monument. Un exemple concret: Le Monument aux Fusillés de Lille. Outre le monument, nous possédons des données complémentaires sur les hommes présents sur le monument (photos de leurs tombes dans un cimetière de Lille...)

Bibliographies

Sites Internet

Informations complémentaires sur votre dépôt

Ce champ libre vous permet de mettre des informations que vous n'avez pu signaler dans les catégories ci-dessus.

\section{Partie 3}

Cette partie est très importante, car elle contient les principaux renseignements et plus particulièrement les médias du monument

Type de monument. 
117 En ouvrant la fenêtre, vous pouvez déterminer le type de monument. Communal, paroissial... Il est possible de cocher plusieurs cases.

118 Médias du monument. La manœuvre doit être effectuée photo par photo, pas de saisie en lots.

119 - Catégorie. Le choix permettra de placer les médias dans les diverses catégories qui apparaîtront sur la fiche en ligne du monument. Les catégories sont: Maquettes/ dessins ; Photographies anciennes (cartes postales la plupart du temps) ; Inscription ; Photographies actuelles; Localisation (document ancien trouvé dans les archives); Noms des morts ; Sources diverses.

* Source. Selon la catégorie, vous indiquez ici: pour Maquettes/Dessins (la provenance et la cote des archives); pour les cartes postales, la provenance (coll. privée, ou cote, si fonds d'archives...); pour les photos actuelles, le nom du photographe et la date de prise de vue.

121 - Description. Une description courte qui peut contenir, par exemple pour les cartes postales, le titre figurant sur la carte ; texte complémentaire relié à la photo (de face, arrière...)

122 -* Fichier. À ce stade vous ajoutez chaque photo et à la fin vous enregistrez.

123 Auteurs

124 -* Métier. Vous choisissez dans le menu déroulant le type de métier (architecte, sculpteur...) ; en fonction de ce choix, vous tapez le nom que vous avez relevé soit sur le monument, soit dans des archives.

125 -* Auteur. Si le nom existe il s'affichera et vous n'aurez qu'à valider le choix, sinon vous tapez le nom complètement et vous enregistrez.

126 - Les médias concernant les auteurs seront à déposer directement sur la fiche auteurs.

127 Produits. Ce terme permet la description guidée selon des catégories prédéfinies.

128 - Type de produit. Architecte, Ornements, Sculptures...

129 - Produit. En fonction du choix d'un type de produit, vous avez une liste de produits correspondant. Si vous choisissez Sculpture/Soldats, le menu produit vous indiquera une liste à choisir.

130 Événements.

131 Cette rubrique texte va nous permettre de renseigner la vie chronologique du monument. Elle est alimentée grâce aux documents d'archives (communales ou départementales ou privées si vous en avez)

132 - * Type Événement. Entrées prédéfinies en fonction du document mis en ligne.

133 - Source Événement. Provenance et cote du document.

134 - Description Événement. Il est possible de retranscrire le texte du document intégralement, si vous en avez le temps.

135 - Date Jour. Choisir le jour.

136 - Date mois. Choisir le mois.

137 - Date année. Mettre l'année. 
139 Après avoir choisi la guerre concernée, remplir avec les noms des morts tels qu'ils sont indiqués sur le monument.

140 - * Commémorations. Choix de la guerre.

141 - Liste des morts. Copie des noms des morts tels qu'ils sont inscrits sur le monument.

142 Pour ce champ, si vous n'avez pas la liste des morts, indiquez au moins la guerre. Si vous laissez ce champ non rempli, la recherche sur 1914-1918 sera faussée. On peut revenir à tout moment sur les fiches incomplètes ensuite.

Et pour terminer, vous pouvez Soumettre le dépôt

ATTENTION, le dépôt ne sera visible sur la base qu'après validation de l'administrateur (ou des administrateurs secondaires dans chaque département, lorsqu'ils auront été choisis).

\section{Soumission via les fiches déjà existantes}

Si la fiche existe déjà et que vous avez des informations complémentaires, vous pouvez SOUMETTRE de nouvelles informations. La procédure est la même, sauf les que les coordonnées du monument existent déjà. Ces compléments d'information sont également possibles pour les fiches auteurs et pour les fiches communes.

\section{Conclusion}

Ce travail sur les monuments aux morts est pour nous tout d'abord un travail de recherche. Il a été conçu pour être utilisable pour tout public. Les chercheurs en histoire de l'art peuvent y contribuer en enrichissant les fiches auteurs; les enseignants en histoire économique peuvent étudier le coût de ces constructions commémoratives; les enseignants du secondaire peuvent l'utiliser avec leurs élèves comme base de travail pédagogique, etc. Lors des collectes Européana dans les archives départementales du Nord, certaines personnes avec qui j'ai échangé ont découvert que leur aïeul avait son nom sur tel ou tel monument de la commune et à chaque fois une certaine émotion se dégageait.

La base va servir également au projet mené par les Rencontres d'Arles ${ }^{40}$. Le grand photographe et cinéaste Raymond Depardon a accepté de parrainer cette opération, notamment en prodiguant un protocole de prise de vue; celui-ci est très proche de celui que nous avions défini. Les participants sont invités à se rendre sur le portail Internet de la Mission du centenaire de la Première Guerre mondiale (http:// monuments.centenaire.org/cartographie/) ou sur le site des Rencontres de la Photographie d'Arles (http://www.rencontres-arles.com/Home) pour prendre connaissance du protocole de prise de vue et y déposer leurs photographies uniquement sous forme numérique, qui peuvent être réalisées à l'aide de tout type d'appareils photographiques (numérique, argentique, smartphone). Il sera remis à tous les participants dont les photographies ont été sélectionnées deux invitations pour visiter l'édition 2014 des Rencontres de la Photographie d'Arles. Ces dépôts permettront également d'enrichir la base nationale des monuments aux morts conçue par l'IRHiS, laboratoire de recherches du CNRS et de l'Université de Lille-3, partenaire de la Mission du centenaire de la Première Guerre mondiale. 
148 L'ensemble des maires de France et responsables d'institutions publiques (transports, administrations, armée...) seront sollicités pour faire remonter les photographies numériques. Ils permettront de solliciter à la fois les dépôts sur la base et de trouver des "volontaires éclairés " pour devenir administrateurs secondaires de cette base. Nous souhaiterions un correspondant-administrateur par département. Il serait chargé de valider les dépôts. Nous pensons faire pour ces correspondants une petite formation dans le courant de l'année, peut-être par vidéoconférence ou Skype.

\section{NOTES}

1. - HARGROVE, June Ellen. «Souviens-toi». Bulletin des Monuments Historiques, $n^{\circ} 124$, janvier 1983, p. 59-65 ; BARCELLINI, Serge et WIEWORKA, Annette. Passant, souviens-toi! Les lieux du souvenir de la Seconde Guerre mondiale en France. Paris : Graphein, 1999.

2. - L'équipe au sein du laboratoire IRHiS-Université de Lille 3 est constituée de Robert Vandenbussche, Martine Aubry, Matthieu de Oliveira, Élise Julien, Sabine Dullin, Jean-Marc Guislin, Matthias Meirlaen (post-doctorant, depuis cette année), d'étudiants (dossiers de licence), des conservateurs des archives départementales du Nord; la base a pris rapidement un très large essor et rassemblé de plus en plus de collaborations extérieures.

3. - Seuls 10 départements ne sont pas représentés à ce jour (4/02/14) : les Hautes-Pyrénées, la Corse du Sud, la Moselle, les Vosges et le Territoire de Belfort, enfin les 5 départements de la région parisienne : Essonne, Hauts-de-Seine, Seine-Saint-Denis, Val-d'Oise et Val-de-Marne.

4. - Vol. 1 : Histoire ; Vol. 2 : Sociologie ; Vol. 3 : Mentalités et idéologies. Paris : FNSP, 1977.

5. - Il confirme ses premières analyses dans son article « Les monuments aux morts ». Les lieux de mémoire. I - La République. NORA, Pierre (dir.). Paris : Gallimard, «Bibliothèque des histoires", 1984, p. 195-225.

6. - RIVÉ, Philippe. Monuments de mémoire : les monuments aux morts de la Première Guerre Mondiale. Paris : Mission permanente aux commémorations et à l'information historique, 1991 ; BECKER, Annette. Les Monuments aux morts. Mémoire de la Grande Guerre. Paris : Errance, 1988.

7. - Le plus connu est sans conteste celui de la commune de Gentioux-Pignerolle (Creuse) qui met en scène un enfant, le poing tendu vers l'inscription "Maudite soit la guerre ». Sur cette thématique, voir l'ouvrage de ROY, Danielle et Pierre. Autour de monuments aux morts pacifistes en France : histoire et présentation d'édifices de la mémoire pacifiste et laïque et évocation de leur actualité : rassemblements de commémoration et d'action contre la guerre. Suresnes: Fédération nationale laïque des Associations des amis des monuments pacifistes, républicains et anticléricaux, 1999.

8. - BECKER, Annette. « Monuments et cérémonies de 1871 aux années vingt ». Dans Monuments de mémoire... op. cit., p. 17-29.

9. - On en trouve par exemple à Bourges, où le monument dédié «Aux enfants du Cher morts pour la patrie 1870-1871» est surmonté de la sculpture de l'« homme taureau » et commémore les combats du $19^{\mathrm{e}}$ régiment de mobiles du Cher contre les Prussiens à Juranville.

10. - BECKER, Jean-Jacques et BERSTEIN, Serge. Victoire et frustrations, 1914-1929. Paris : Le Seuil, «Nouvelle histoire de la France contemporaine - 10», 1990, chap. 7 : «L'ombre portée de la guerre ", p. 155-178; DUPÂQUIER, Jacques (dir.). Histoire de la population française, Vol. 4 : de 1914 à nos jours. Paris : PUF, 1988. 
11. - DALISSON, Rémi. 11 novembre. Du souvenir à la mémoire. Paris : Armand Colin, 2013.

12. - BECKER, Annette. «Les monuments aux morts, miroirs de la Grande Guerre ». Historiens et Géographes, $\mathrm{n}^{\circ}$ spécial 321, p. 293-296.

13. - Séraphin Monge, le héros de La maison assassinée, de Pierre Magnan [Denoël, 1983 ; porté à l'écran par G. Lautner en 1988] rentre de la guerre et trouve son nom gravé sur le monument aux morts, parce que « ça en faisait un de plus que le village voisin ».

14. - Dans son roman Au revoir là-haut [Albin Michel, 2013], récemment récompensé du Prix Goncourt, Pierre Lemaître imagine d'ailleurs une vaste escroquerie visant à lancer une souscription nationale auprès des municipalités pour l'achat de monuments aux morts qui ne seront jamais érigés, mais dont ses héros tirent un immense profit.

15. - En attendant de lire la thèse en cours de Christina Théodosiou, Le 11 novembre pendant l'entredeux-guerres, (Paris I, Ch. Charle dir.), on renvoie aux deux articles disponibles de l'auteur: "Symbolic narratives and the legacy of the Great War: the celebration of Armistice Day in France in the 1920s ». First World War Studies, vol. 1, n 2, 2010, p. 185-198 et « La mobilisation des morts : culte du souvenir et culture de guerre en France pendant la Grande Guerre ». Revue LISA/ LISA e-journal [En ligne], Vol.X - $\mathrm{n}^{\circ} 1 \mid 2012$, mis en ligne le 12 mars 2012, consulté le 20 janvier 2014. URL : http://lisa.revues.org/4844 ; DOI : 10.4000/lisa.4844.

16. - Dans l'état actuel des relevés, appartiennent à cette catégorie les communes de Narbief (Doubs), Palazingues (Corrèze), Peuvillers (Meuse), Thierville (Eure), Nampty et Hervilly (Somme), Le Manoir et Beuzeville-au-Plain (Manche).

17. - Le maire de Beuzeville-au-Plain signale qu' « un soldat a été blessé en sauvant un officier. Il s'en est sorti. ». Le Point, 10 novembre 2011.

18. - DELAPORTE, Sophie. Les Gueules cassées : les blessés de la face de la Grande Guerre. Paris : Noêsis, 1996 ; TISON, Stéphane. "Traumatisme de guerre et commémorations ». Guerres mondiales et conflits contemporains, 4/2004 ( $\left.\mathrm{n}^{\circ} 216\right)$, p. 5-29.

19. - ANDRIVON-MILTON, Sabine. La Martinique et la Grande Guerre. Paris : L'Harmattan, 2005 ; LOUIS, Patrick. « Les « poilus » martiniquais réhabilités ». Le Monde, 11 novembre 2006.

20. - KIDD, William. Les monuments aux morts mosellans : de 1870 à nos jours. Metz : Éd. Serpenoise, 1999 ; NIESS, Alexandre et PHILIPPOT, Jacques (ill.). Cimetières militaires et monuments aux morts de la Grande Guerre, Marne. Langres: D. Guéniot, 2005 ; WAGNER, Gilbert. Lieux de mémoire dans le Haut-Rhin: les monuments aux morts, les stèles et plaques de mémoire, les nécropoles françaises, les cimetières militaires allemands, le cimetière roumain. Sl: Conseil général du Haut-Rhin, 2012; BONNET, Claude. Les monuments aux morts dans le département du Doubs : guerre 1914-1918 : le culte du souvenir ou la mémoire collective de la nation. Franois: Empreinte Éd., 1998; SIFFERT, Nicole et COAT, Paul. Les monuments aux morts du Territoire de Belfort. Colmar : Do Bentzinger, 2001.

21. - LUIRARD, Monique. La France et ses morts. Les monuments commémoratifs dans la Loire. SaintÉtienne : Centre interdisciplinaire d'études et de recherches sur les structures régionales, 1977 ; JAM, Alain et GRAMBIN, Marc. Mémoires de vies: canton d'Allevard, département de l'Isère: les monuments aux morts de la Grande Guerre: avec mémorandum \& autres documents. Le Moutaret: Autrefois pour tous éd., 2006 ; GIROUD, Jean, MICHEL, Raymond et Maryse. Les monuments aux morts de la guerre 1914-1918 dans le Vaucluse. L'Isle-sur-Sorgue : Éd. Scriba, 1991; MATTONNEVASTEL, Sylvie et MEISSONNIER, Georges. L'art et la mémoire de 1914-1918 dans le Var. Toulon: Commission départementale de l'information historique pour la paix, 1998; PELLEGRINETTI, Jean-Paul et RAVIS-GIORDANI, Georges. «Les monuments aux morts de la première guerre mondiale en Corse ». Cahiers de la Méditerranée [En ligne], 81 | 2010, mis en ligne le 15 juin 2011.

22. - MOISAN, Hervé. Sentinelles de pierre. Les monuments aux morts de la guerre de 1914-1918 dans la Nièvre, préface d'A. Prost. Saint-Pourçain-sur-Sioule : Bleu autour, 1999; AURÉJAC, Francis et WECK, Arlette. Mourir pour la patrie : l'hommage des creusois : monuments et cérémonies: 1915-1939. Guéret: Archives départementales de la Creuse, 1998 ; CHALINE, Nadine-Josette et MOULINET, 
Daniel. Gardiens de la mémoire. Les monuments aux morts de la Grande Guerre dans l'Allier. Yzeure : Amis du Patrimoine Religieux en Bourbonnais, 2008.

23. - VERGEZ, François. Monuments aux morts et mouvements commémoratifs de la guerre 14-18 en Béarn et Pays Basque. Pau : Office national des anciens combattants et victimes de guerre, service départemental des Pyrénées-Atlantiques, 2008 ; ALÉGRIA, Ludivine. Monuments aux morts de la Grande Guerre dans les Landes. Mont-de-Marsan : Conseil général des Landes, 2004.

24. - BOURDIN, Gérard. Les monuments aux morts dans l'Orne : pour le deuil ou pour l'exemple? Guerre du XIXe siècle, guerre 1870-1871, guerre 1914-1918. Flers : «Le Pays bas-normand », 1992 ; GILQUIN, Jean-Claude. La pierre du souvenir : les monuments aux morts de la guerre 1914-1918 dans le Val d'Oise. Pontoise : Éd. du Valhermeil, 1994 ; LAFAGES-VITALIS, Mélitza, DELUNEAU, Anaïs et GEOFFROY, Maïté. Les monuments aux morts de la Grande Guerre 1914-1918 en Guadeloupe. Les Abymes : CRDP Guadeloupe, 2006.

25. - À la croisée des chemins : les monuments aux morts de l'Aisne. Wambrechies : Bayard service Éd., 2011.

26. - GUERRINI, Dominique et Jean-Étienne. La Somme à ses enfants. Amiens : Association pour la généralisation de l'inventaire régional en Picardie, 1994 ; DELFORGE, Cécile. Les monuments aux morts de la Grande Guerre dans le département de la Somme. Maîtrise d'Histoire contemporaine, Université de Lille 3, 1994.

27. - Quelques travaux ont déjà été réalisés et recensés comme par exemple: GRAILLES, Bénédicte. Mémoires de pierre : les monuments aux morts de la Première Guerre Mondiale dans le Pas-deCalais. Catalogue d'exposition, Arras : Archives départementales du Pas-de-Calais, 1992 et VAREZ, Benoît. Les monuments aux morts et les cimetières militaires commémoratifs de la première guerre mondiale dans le canton de La Bassée. Mémoire de maîtrise, Université de Lille 3, 2004.

28. - La «zone rouge ", où les destructions sont totales, y est particulièrement importante ; on estime ainsi que le département du Nord a subi à lui seul plus de $60 \%$ des dommages de guerre du territoire national.

29. - NIVET, Philippe. La France occupée, 1914-1918. Paris : A. Colin, 2011.

30. - C'est par exemple le cas des trois communes nordistes d'Hargnies, d'Iwuy et du CateauCambrésis.

31. - HILAIRE, Yves-Marie et alii. Atlas électoral Nord-Pas-de-Calais, 1 : 1876-1936, Troisième République. Villeneuve d'Ascq: Publications de l'Université de Lille 3, 1977 et plus largement : SALMON, Frédéric. Atlas électoral de la France, 1848-2001. Paris : Le Seuil, 2001.

32. - La situation est tout à fait comparable à Wambrechies (Nord).

33. - Une chrétienté au XIX ${ }^{e}$ siècle? La vie religieuse des populations du diocèse d'Arras, 1840-1914. Villeneuve-d'Ascq : Université de Lille III, 1977, 2 vol.

34. - Soit quelques mois avant celles de 1920 signalées par A. Prost.

35. - CAPDEVILA, Luc et VOLDMAN, Danielle. «Rituels funéraires de sociétés en guerre (1914-1945) ». La violence de guerre, 1914-1945. Bruxelles : Complexe-IHTP, 2002, p. 289-311.

36. - BRANCHE, Raphaëlle et THÉNAULT, Sylvie (dir.). La France en guerre 1954-1962 : expériences métropolitaines de la guerre d'indépendance algérienne. Paris : Éd. Autrement, 2008.

37. - En 2012, 82 soldats français avaient trouvé la mort en Afghanistan ; en 2013, 7 militaires sont tombés au Mali et 2 autres ont perdu la vie début 2014 en République centrafricaine. Armée d'aujourd'hui, n 385, décembre 2013-janvier 2014, p. 44.

38. - PEF. Zappe la guerre. Paris : Rue du Monde, 2005. Il s'inspire explicitement de l'expérience de ses grands-pères et grand-oncle, auxquels l'ouvrage est dédié.

39. - Depuis l'écriture de cet article, la base a considérablement évolué. Nous sommes aujourd'hui (10 septembre 2014) à 8624 monuments ( 8522 pour la France et 102 pour la Belgique).

40. - Les Rencontres de la photographie sont le grand rendez-vous international des spécialistes de la photographie la première semaine de juillet, largement ouvertes au public tout l'été ; elles 
ont accueilli près de 100000 visiteurs en 2013, provenant de toute la France et pour $15 \%$ de l'étranger. La "Rentrée en images" en septembre reçoit chaque année la visite de plus de 10000 élèves, 300 classes de 8 académies provenant de toutes les régions françaises.

\section{ABSTRACTS}

After the First World War, families, local authorities and the whole nation wanted to pay homage to 'the heroes fallen for France'. From 1919 on war memorials appeared in practically each French commune, with commemorative messages that could vary according to the location, the date of construction or the number of war dead to be remembered. Like the statues themselves, the wording of the texts engraved, is a subject worth studying. The database set up by the research department IRHIS (UMR 8529) and given the label of the French mission for the First World War centenary commemorations aims to draw up an exhaustive inventory of these war memorials, thought to number about 40,000 in all. The database also sets out to provide illustrations with historic and contemporary pictures, press cuttings, archival documents, geolocalisation data and texts concerning iconographic or economic aspects of the monument. The analysis of the results of this database for the Nord-Pas-de-Calais region has produced some surprising results and the first conclusions of this on-going project already allow for some interregional and national comparisons.

$\mathrm{Au}$ lendemain de la première guerre mondiale, les familles, les municipalités comme la nation toute entière entendent rendre hommage aux « héros tombés pour la France ». Dès 1919, on voit ainsi des monuments aux morts apparaître dans chaque commune, ou presque, et porter un message commémoratif qui peut varier selon le lieu, le moment de son érection ou encore le nombre de morts. En ce sens, le choix du texte gravé comme celui de la statue retenue deviennent des objets d'étude. La base de données portée par l'IRHiS (UMR 8529) et labellisée par la Mission du centenaire, entend en premier lieu recenser l'ensemble de ces monuments, évalués à 40000 au total, mais surtout proposer un outil associant photos (d'époque comme d'aujourd'hui), documents tirés des archives ou de la presse, géo-localisation et enfin caractéristiques iconographiques, textuelles ou économiques des monuments. L'analyse de la situation du Nord-Pas-de-Calais, aux résultats inédits, permet de disposer de premières conclusions à comparer aux résultats inter-régionaux puis nationaux au terme du processus de collecte.

\section{INDEX}

Mots-clés: monuments aux morts ; base de données ; commémoration ; 1914-1918 ; Nord-Pas-deCalais ; France

Keywords: War memorials, database, commemoration, 1914-1918, Nord-Pas-de-Calais, France 


\section{AUTHORS}

\section{MARTINE AUBRY}

Ingénieur de Recherche, Responsable Valorisation de la Recherche, Cellule Nouvelles

Technologies, Université de Lille (Lille 3) - IRHiS (UMR 8529) martine.aubry@univ-lille3.fr

\section{MATTHIEU DE OLIVEIRA}

maître de conférences en histoire contemporaine IRHiS (UMR 8529) matthieu.deoliveira@univ-

lille3.fr 